\title{
Qualitative properties for a pseudo-parabolic equation with nonlocal reaction term
}

\author{
Yadong Zheng ${ }^{1}$ and Zhong Bo Fang ${ }^{1 *}$ (D)
}

\section{"Correspondence:}

fangzb7777@hotmail.com

'School of Mathematical Sciences,

Ocean University of China, Qingdao,

P.R. China

\section{照 Springer}

\begin{abstract}
This paper deals with the qualitative properties of solutions for null Neumann initial boundary value problem to a nonlocal pseudo-parabolic equation in the sense of $H^{\prime}(\Omega)$-norm. We establish sufficient conditions to guarantee that the solution with initial energy exists globally or blows up at finite time under an appropriate range of parameters. Moreover, life span of the blow-up solution, decay rate of the global solution, and growth estimate are derived.
\end{abstract}

MSC: 35511;35B40; 35B44

Keywords: Pseudo-parabolic equation; Nonlocal reaction term; Blow-up; Life span; Asymptotic behavior

\section{Introduction}

We consider a pseudo-parabolic equation with nonlocal reaction term

$$
u_{t}-k \Delta u_{t}-\Delta u=|u|^{p}-\frac{1}{|\Omega|} \int_{\Omega}|u|^{p} d x, \quad(x, t) \in \Omega \times\left(0, t^{*}\right),
$$

subject to null Neumann boundary and initial conditions

$$
\begin{aligned}
& \frac{\partial u}{\partial n}=0, \quad(x, t) \in \partial \Omega \times\left(0, t^{*}\right), \\
& u(x, 0)=u_{0}(x), \quad x \in \Omega,
\end{aligned}
$$

where $\Omega \subset \mathbb{R}^{N}(N \geq 1)$ is a bounded domain with smooth boundary $\partial \Omega, p>0$, $t^{*}$ is the maximal existence time of solutions, the initial data $u_{0}(x) \in H^{1}(\Omega) \cap L^{p+1}(\Omega)$ satisfies $u_{0}(x) \not \equiv 0$ and $\int_{\Omega} u_{0}(x) d x=0$.

Nonlocal pseudo-parabolic equation like (1.1) describes a variety of physical phenomena, such as the seepage of homogeneous fluids through a fissured rock, the unidirectional propagation of nonlinear, dispersive, long waves, heat conduction problems with thermodynamic temperature and conduction temperature, and the analysis of nonstationary processes in semiconductors in the presence of sources (see [1-5]). In particular, equation (1.1) is a possible model for populations with the tendency to form groups, where

(c) The Author(s) 2019. This article is distributed under the terms of the Creative Commons Attribution 4.0 International License (http://creativecommons.org/licenses/by/4.0/), which permits unrestricted use, distribution, and reproduction in any medium, provided you give appropriate credit to the original author(s) and the source, provide a link to the Creative Commons license, and indicate if changes were made. 
$u(x, t)$ represents the density of the species at position $x$ and time $t$, and the reaction term $|u|^{p}-\frac{1}{|\Omega|} \int_{\Omega}|u|^{p} d x$ is considered as the rate of reproduction. Nonlocal term $\int_{\Omega} u^{p} d x$ is a way to express that the evolution of the species in a point of space depends not only on nearby density but also on the mean value of the total amount of species due to the effects of spatial inhomogeneity (see [6-9]). Nonlocal reaction term can also describe Darwinian evolution of a structured population density or the behaviors of cancer cells with therapy (see [10-12]).

In this paper, we are interested in studying the qualitative properties of solutions for a pseudo-parabolic equation (1.1) with nonlocal reaction term $|u|^{p}-\frac{1}{|\Omega|} \int_{\Omega}|u|^{p} d x$ under the null Neumann boundary condition, which has a typical structure of mass conservation. Let us recall some backgrounds firstly. When $k=0$, (1.1) reduces to a classical heat equation, and there have been many good results (see [13-21] and the references therein). For instance, Budd et al. [13] considered the following semilinear heat equation with nonlocal reaction term:

$$
u_{t}-u_{x x}=u^{2}-\int_{0}^{1} u^{2} d x, \quad(x, t) \in(0,1) \times\left(0, t^{*}\right)
$$

they derived the existence of a trivial steady solution. Moreover, the finite time blow-up phenomena for sufficiently large initial data were proved by using a comparison principle in a Fourier space. The above equation is also related to the Navier-Stokes equation on an infinite slab (see [14]). Hu and Yin [15] studied the nonlocal semilinear parabolic equation in a higher dimensional space

$$
u_{t}-\Delta u=|u|^{p-1} u-\frac{1}{|\Omega|} \int_{\Omega}|u|^{p-1} u d x, \quad(x, t) \in \Omega \times\left(0, t^{*}\right)
$$

for the superlinear case $(p>1)$, they established a result of blow-up with negative initial energy by using a convexity argument. Later, Gao and Han [16] extended the result to the case of positive initial energy. Soufi et al. [17] investigated a similar problem and established a relation between the finite time blow-up of solutions and the negativity of initial energy for $1<p \leq 2$ by using gamma-convergence argument. They also conjectured that the relation might hold for all $p>1$, a positive answer to which was given by Jazar in [18]. In addition, Niculescu and Roventa [19] considered a more general initial boundary value problem of nonlocal semilinear parabolic equation given by

$$
u_{t}-\Delta u=f(|u|)-\frac{1}{|\Omega|} \int_{\Omega} f(|u|) d x, \quad(x, t) \in \Omega \times\left(0, t^{*}\right)
$$

with the null Neumann boundary condition. They established a blow-up result when $f(|u|)$ belongs to a large class of nonlinearities and the initial energy was non-positive by using the convexity method. Recently, concerning the research on the blow-up rate and convergence results of solutions for the following reaction-diffusion model in one-dimensional space

$$
u_{t}-u_{x x}=|u|^{p}-\frac{1}{2 \pi} \int_{0}^{2 \pi}|u|^{p} d x, \quad(x, t) \in(0,2 \pi) \times\left(0, t^{*}\right)
$$


in the sense of suitable norm, one can refer to [20, 21]. Yan and Yang [22] studied the Neumann initial boundary value problem of semilinear parabolic equation with logarithmic nonlinearity

$$
u_{t}-\Delta u=u \log |u|-\frac{1}{|\Omega|} \int_{\Omega} u \log |u| d x, \quad(x, t) \in \Omega \times\left(0, t^{*}\right) .
$$

By using the logarithmic Sobolev inequality and energy estimate methods, they proved that the solution with negative initial energy blows up at infinity and the solution with positive initial energy is non-extinct. Besides, for the recent advances in thin-film equation and quasilinear equation with nonlocal reaction term, we refer to [23-27].

When $k \neq 0$, most of the research concentrated on studying qualitative properties to the Cauchy problem and the Dirichlet initial boundary value problem, but much less effort has been devoted to the Neumann problem. For the equation with local source terms $u_{t}-k \Delta u_{t}-\Delta u=u^{p}$, Cao et al. [28] studied its Cauchy problem and got the critical exponent of global existence and the critical Fujita exponent by the integral representation and the contraction-mapping principle. They also proved that the appearance of the third order term $\Delta u_{t}$ has no effect on the critical Fujita exponent for the classical heat equation. $\mathrm{Xu}$ and $\mathrm{Su}$ [29] investigated the global existence, blow-up, and asymptotic behavior of solutions to the Dirichlet initial boundary value problem with arbitrary initial energy by using the potential well method. Later on, Luo [30] estimated the life span of solutions with negative initial energy, and the decay estimate of the global solution was established. Moreover, $\mathrm{Xu}$ and Zhou [31] extended the results in [30] to the case of nonnegative initial energy. For pseudo-parabolic equations with nonlocal source terms, Yang and Liang [32] discussed a nonlocal semilinear pseudo-parabolic equation

$$
u_{t}-\Delta u_{t}-\Delta u=u^{p}(x, t) \int_{\Omega} k(x, y) u^{p+1}(y, t) d y, \quad(x, t) \in \Omega \times\left(0, t^{*}\right),
$$

under null Dirichlet boundary condition, they obtained the upper and lower bounds of the blow-up time and the exponential decay estimates of the global solution. However, they did not consider the nonlocal reaction terms of the same mass conservation structure as our model (1.1).

Motivated by the above works, the study of qualitative properties of solutions for nonlocal pseudo-parabolic model (1.1)-(1.3) in the $H^{1}(\Omega)$-norm has not been proceeded yet. Our main difficulties lie in finding the influence of the third order term $\Delta u_{t}$ and nonlocal reaction term on the qualitative properties, and the solutions may be sign-changing. Using the method of energy functional and the convexity technique, we establish sufficient conditions to guarantee the solution with initial energy exists globally or blows up in finite time under appropriate norms. Moreover, life span of the blow-up solution, decay rate of the global solution, and growth estimate are derived.

The rest of our paper is organized as follows. In Sect. 2, we introduce some preliminaries and main results. Then we give the proofs of the main results in Sect. 3.

For simplicity, we take $k=1$. All the discussions and results obtained in this paper are valid for any positive number $k$. 


\section{Preliminaries and main results}

In this section, we introduce some notations, functionals, sets, and main results. For convenience, we denote by $\|\cdot\|_{r}$ and $\|\cdot\|_{H^{1}}=\left(\|\cdot\|_{2}^{2}+\|\nabla \cdot\|_{2}^{2}\right)^{\frac{1}{2}}$ the norms of $L^{r}(\Omega)$ for $1 \leq r \leq+\infty$ and $H^{1}(\Omega)$, respectively. Meanwhile, we introduce the following space:

$$
\begin{aligned}
& X:=L^{\infty}(\Omega) \text { or } C(\bar{\Omega}), \\
& H:=\left\{u \in H^{1}(\Omega):\left.\frac{\partial u}{\partial n}\right|_{\partial \Omega}=0, \int_{\Omega} u d x=0\right\},
\end{aligned}
$$

and the weak continuous function spaces $C_{w}^{0}\left(0, T ; L^{2}(\Omega)\right)$ on $(0, T)$.

Note that our model satisfies the conservation of mass, and the initial condition implies that

$$
\int_{\Omega} u(x, t) d x=\int_{\Omega} u_{0}(x) d x=0
$$

and hence, there holds Poincaré's inequality in $H^{1}(\Omega)$

$$
\|u\|_{2}^{2} \leq C_{p}\|\nabla u\|_{2}^{2}
$$

where $C_{p}=C_{p}(\Omega, 2)$ is the Poincaré's inequality constant in $H^{1}(\Omega)$.

The local existence of the solutions for sublinear and linear cases $(0<p \leq 1)$ can be obtained via the Galerkin approximation method [24] when $u_{0}(x) \in H$, then problem (1.1)(1.3) has solutions satisfying

$$
u \in L^{\infty}(0, T ; H) \cap C_{w}^{0}\left(0, T ; L^{2}(\Omega)\right), \quad u_{t} \in L^{2}(0, T ; H)
$$

For superlinear cases $(p>1)$, we can obtain the existence and uniqueness of solutions for problem (1.1)-(1.3) directly according to [8, Theorem 1 and Proposition 3] when $u_{0}(x) \in X$.

Next, we define two functionals $J(u)$ and $I(u)$ by

$$
\begin{aligned}
& J(u):=\frac{1}{2} \int_{\Omega}|\nabla u|^{2} d x-\frac{1}{p+1} \int_{\Omega}|u|^{p} u d x, \\
& I(u):=\int_{\Omega}|\nabla u|^{2} d x-\int_{\Omega}|u|^{p} u d x .
\end{aligned}
$$

Furthermore, it is not difficult to obtain the following equality:

$$
\int_{0}^{t}\left\|u_{\tau}\right\|_{H^{1}}^{2} d \tau+J(u(t))=J\left(u_{0}\right)
$$

and then $J(u(t))$ is nonincreasing with respect to $t$.

Now we state our main results.

Theorem 1 (Superlinear case) Assume that $p>1$ and $u_{0}(x) \in H^{1}(\Omega) \cap L^{p+1}(\Omega)$. If one of the following two conditions holds: 
(i) $J\left(u_{0}\right)<0$,

(ii) $0 \leq J\left(u_{0}\right)<\frac{p-1}{2(p+1)\left(C_{p}+1\right)}\left\|u_{0}\right\|_{H^{1}}^{2}$,

then the solution $u(x, t)$ to problem (1.1)-(1.3) blows up at finite time $t^{*}$ in the sense of $H^{1}(\Omega)$-norm. Moreover, $t^{*}$ can be estimated by (3.8) and (3.22), respectively, i.e.,

$$
t^{*}<\frac{\left\|u_{0}\right\|_{H^{1}}^{2}}{\left(1-p^{2}\right) J\left(u_{0}\right)}
$$

and

$$
t^{*} \leq \frac{8(p+1)\left(C_{p}+1\right)\left\|u_{0}\right\|_{H^{1}}^{2}}{(p-1)^{2}\left[(p-1)\left\|u_{0}\right\|_{H^{1}}^{2}-2(p+1)\left(C_{p}+1\right) J\left(u_{0}\right)\right]}
$$

Furthermore, if p satisfies

$$
1<p<+\infty, \quad N=1,2 ; \quad 1<p \leq \frac{N+2}{N-2}, \quad N \geq 3
$$

then the lower bound of $t^{*}$ can be estimated by (3.24), i.e.,

$$
t^{*} \geq \frac{1}{C_{s}^{p+1}(p-1)\left\|u_{0}\right\|_{H^{1}}^{p-1}}
$$

where $C_{s}=C_{s}(\Omega, p)$ is the Sobolev embedding constant from $H^{1}(\Omega)$ to $L^{p+1}(\Omega)$.

Remark 1 The upper bound of the blow-up time obtained from (3.8) and (3.22) is larger than the lower bound from (3.24). In fact, due to $p>1$, we can easily get the following two inequalities by Poincaré's inequality and Sobolev's inequality:

$$
\begin{aligned}
& \frac{1}{2} \int_{\Omega}\left|\nabla u_{0}\right|^{2} d x+\frac{1}{p+1} C_{s}^{p+1}\left\|u_{0}\right\|_{H^{1}}^{p+1} \geq \frac{1}{p+1} \int_{\Omega}\left|u_{0}\right|^{p} u_{0} d x \\
& \frac{1}{2} \int_{\Omega}\left|\nabla u_{0}\right|^{2} d x-\frac{(p-1)\left\|u_{0}\right\|_{H^{1}}^{2}}{2(p+1)\left(C_{p}+1\right)}+\frac{4 C_{s}^{p+1}\left\|u_{0}\right\|_{H^{1}}^{p+1}}{p-1} \geq \frac{1}{p+1} \int_{\Omega}\left|u_{0}\right|^{p} u_{0} d x .
\end{aligned}
$$

Therefore, the life spans of the blow-up solution are given by

$$
\frac{1}{C_{s}^{p+1}(p-1)\left\|u_{0}\right\|_{H^{1}}^{p-1}} \leq t^{*}<\frac{\left\|u_{0}\right\|_{H^{1}}^{2}}{\left(1-p^{2}\right) J\left(u_{0}\right)}
$$

and

$$
\frac{1}{C_{s}^{p+1}(p-1)\left\|u_{0}\right\|_{H^{1}}^{p-1}} \leq t^{*} \leq \frac{8(p+1)\left(C_{p}+1\right)\left\|u_{0}\right\|_{H^{1}}^{2}}{(p-1)^{2}\left[(p-1)\left\|u_{0}\right\|_{H^{1}}^{2}-2(p+1)\left(C_{p}+1\right) J\left(u_{0}\right)\right]}
$$

respectively.

Corollary 1 Assume that $p>1$ and $u_{0}(x) \in H^{1}(\Omega) \cap L^{p+1}(\Omega)$. Then the solution $u(x, t)$ to problem (1.1)-(1.3) blows up at finite time $t^{*}$ in the sense of $H^{1}(\Omega)$-norm with arbitrary initial energy. 
Theorem 2 (Sublinear case) Assume that $0<p<1$ and $u_{0}(x) \in H^{1}(\Omega) \cap L^{p+1}(\Omega)$. Then the solution $u(x, t)$ to problem (1.1)-(1.3) is global. Moreover, if $J\left(u_{0}\right)<0$ and $\left\|u_{0}\right\|_{H^{1}}^{2}>$ $\frac{\left(C_{p}+1\right) \chi(\varepsilon)|\Omega|}{2-C_{p} \epsilon}$, then $u(x, t)$ decays and is positive in the sense of $H^{1}(\Omega)$-norm and $W^{1, s}(\Omega)$ norm $(\forall s>1)$, respectively, and we have the following estimates:

$$
\|u(t)\|_{H^{1}}^{2} \leq\left(\left\|u_{0}\right\|_{H^{1}}^{2}-\frac{\left(C_{p}+1\right) \chi(\varepsilon)|\Omega|}{2-C_{p} \varepsilon}\right) \exp \left\{-\frac{2-C_{p} \varepsilon}{C_{p}+1} t\right\}+\frac{\left(C_{p}+1\right) \chi(\varepsilon)|\Omega|}{2-C_{p} \varepsilon}
$$

where $0<\varepsilon<\frac{2}{C_{p}}, \chi(\varepsilon)=\frac{1-p}{2}\left(\frac{p+1}{\varepsilon}\right)^{\frac{1}{1-p}}$.

Theorem 3 (Linear case) Assume that $0<p<1$ and $u_{0}(x) \in H^{1}(\Omega) \cap L^{p+1}(\Omega)$. If $J\left(u_{0}\right)<0$, then the solution $u(x, t)$ to problem (1.1)-(1.3) grows within $\left(0, t^{*}\right)$ in the sense of $H^{1}(\Omega)$ norm, and we have

$$
\|u(t)\|_{H^{1}}^{2} \geq\left\|u_{0}\right\|_{H^{1}}^{2} \exp \left\{\frac{-4 J\left(u_{0}\right)}{\left\|u_{0}\right\|_{H^{1}}^{2}} t\right\} .
$$

\section{Proofs of main results}

In this section, we prove our main results in detail.

Proof of Theorem 1 (i) For the case of $J\left(u_{0}\right)<0$, we define the functions

$$
\varphi(t):=\|u\|_{H^{1}}^{2}, \quad \psi(t):=-2(p+1) J(u) .
$$

Firstly, multiplying both sides of equation (1.1) by $u(x, t)$ and using Green's formula, we derive

$$
\varphi^{\prime}(t)=-2 I(u)=-2 \int_{\Omega}|\nabla u|^{2} d x+2 \int_{\Omega}|u|^{p} u d x
$$

Since $p>1$, we have

$$
\psi(t)=-(p+1) \int_{\Omega}|\nabla u|^{2} d x+2 \int_{\Omega}|u|^{p} u d x<\varphi^{\prime}(t) .
$$

Secondly, multiplying both sides of equation (1.1) by $u_{t}(x, t)$ and using Green's formula, we obtain

$$
\int_{\Omega}\left|u_{t}\right|^{2} d x+\int_{\Omega}\left|\nabla u_{t}\right|^{2} d x=-\int_{\Omega} \nabla u \cdot \nabla u_{t} d x+\int_{\Omega}|u|^{p} u_{t} d x .
$$

Differentiating $\psi(t)$ and utilizing (3.3), it yields

$$
\psi^{\prime}(t)=2(p+1) \int_{\Omega}\left(\left|u_{t}\right|^{2}+\left|\nabla u_{t}\right|^{2}\right) d x>0
$$

By (3.4), the fact $J\left(u_{0}\right)<0$, and the definition of $\psi(t)$, it follows that $\psi(t)>0$ for any $t \in$ $\left[0, t^{*}\right)$. 
Afterwards, combining (3.2), (3.4), $\psi(t)>0$, and the Cauchy-Schwarz inequality, we conclude

$$
\begin{aligned}
\varphi(t) \psi^{\prime}(t) & =2(p+1)\left[\int_{\Omega}\left(|u|^{2}+|\nabla u|^{2}\right) d x\right]\left[\int_{\Omega}\left(\left|u_{t}\right|^{2}+\left|\nabla u_{t}\right|^{2}\right) d x\right] \\
& \geq 2(p+1)\left[\int_{\Omega}\left(u u_{t}+\nabla u \cdot \nabla u_{t}\right) d x\right]^{2} \\
& =\frac{p+1}{2}\left[\varphi^{\prime}(t)\right]^{2}>\frac{p+1}{2} \varphi^{\prime}(t) \psi(t),
\end{aligned}
$$

which implies

$$
\frac{\psi^{\prime}(t)}{\psi(t)}>\frac{p+1}{2} \frac{\varphi^{\prime}(t)}{\varphi(t)}
$$

Integrating (3.6) from 0 to $t$, one can see that

$$
\psi(t)>\frac{\psi(0)}{(\varphi(0))^{\frac{p+1}{2}}}(\varphi(t))^{\frac{p+1}{2}}
$$

then by (3.2) we get

$$
\frac{\varphi^{\prime}(t)}{(\varphi(t))^{\frac{p+1}{2}}}>\frac{\psi(0)}{(\varphi(0))^{\frac{p+1}{2}}} .
$$

Integrating (3.7) from 0 to $t$, we deduce

$$
\varphi(t)>\left[(\varphi(0))^{-\frac{p-1}{2}}-\frac{p-1}{2} \frac{\psi(0)}{(\varphi(0))^{\frac{p+1}{2}}} t\right]^{-\frac{2}{p-1}}
$$

which implies that $\varphi(t)$ blows up at some finite time $t^{*}$; what is more, we have

$$
t^{*}<\frac{\left\|u_{0}\right\|_{H^{1}}^{2}}{\left(1-p^{2}\right) J\left(u_{0}\right)} .
$$

(ii) For the case of $0 \leq J\left(u_{0}\right)<\frac{p-1}{2(p+1)\left(C_{p}+1\right)}\left\|u_{0}\right\|_{H^{1}}^{2}$, we may also assume $J(u(t)) \geq 0$ for all $t \in\left[0, t^{*}\right)$. In fact, if there exists $t_{0}$ such that $J\left(u\left(t_{0}\right)\right)<0$, then the solution of problem (1.1)-(1.3) will blow up at finite time according to (i) with $t_{0}$ as the initial time.

Now, we suppose that the solution $u(x, t)$ is global. Then we have

$$
\int_{0}^{t}\left\|u_{\tau}\right\|_{H^{1}} d \tau \geq\left\|\int_{0}^{t} u_{\tau} d \tau\right\|_{H^{1}}=\left\|u(t)-u_{0}\right\|_{H^{1}} \geq\|u(t)\|_{H^{1}}-\left\|u_{0}\right\|_{H^{1}} .
$$

Combining (2.2), (3.9), and Hölder's inequality, we obtain

$$
\begin{aligned}
\|u(t)\|_{H^{1}} & \leq\left\|u_{0}\right\|_{H^{1}}+\int_{0}^{t}\left\|u_{\tau}\right\|_{H^{1}} d \tau \leq\left\|u_{0}\right\|_{H^{1}}+t^{\frac{1}{2}}\left(\int_{0}^{t}\left\|u_{\tau}\right\|_{H^{1}}^{2} d \tau\right)^{\frac{1}{2}} \\
& =\left\|u_{0}\right\|_{H^{1}}+t^{\frac{1}{2}}\left(J\left(u_{0}\right)-J(u(t))\right)^{\frac{1}{2}} \leq\left\|u_{0}\right\|_{H^{1}}+t^{\frac{1}{2}}\left(J\left(u_{0}\right)\right)^{\frac{1}{2}} .
\end{aligned}
$$


On the other hand, by direct calculation and Poincaré's inequality (2.1), one can see that

$$
\begin{aligned}
\frac{d}{d t}\left(\frac{1}{2}\|u(t)\|_{H^{1}}^{2}\right) & =-I(u(t))=-(p+1) J(u(t))+\frac{p-1}{2}\|\nabla u(t)\|_{2}^{2} \\
& =-(p+1) J(u(t))+\frac{(p-1) C_{p}}{2\left(C_{p}+1\right)}\|\nabla u(t)\|_{2}^{2}+\frac{p-1}{2\left(C_{p}+1\right)}\|\nabla u(t)\|_{2}^{2} \\
& \geq-(p+1) J(u(t))+\frac{(p-1) C_{p}}{2\left(C_{p}+1\right)}\|u(t)\|_{2}^{2}+\frac{p-1}{2\left(C_{p}+1\right)}\|\nabla u(t)\|_{2}^{2} \\
& =\frac{p-1}{C_{p}+1}\left[-\frac{\left(C_{p}+1\right)(p+1)}{p-1} J(u(t))+\frac{1}{2}\|u(t)\|_{H^{1}}^{2}\right]
\end{aligned}
$$

Since $\frac{d}{d t} J(u(t)) \leq 0$, then by (3.11) we easily get

$$
\begin{aligned}
& \frac{d}{d t}\left[-\frac{\left(C_{p}+1\right)(p+1)}{p-1} J(u(t))+\frac{1}{2}\|u(t)\|_{H^{1}}^{2}\right] \\
& \quad \geq \frac{p-1}{C_{p}+1}\left[-\frac{\left(C_{p}+1\right)(p+1)}{p-1} J(u(t))+\frac{1}{2}\|u(t)\|_{H^{1}}^{2}\right] .
\end{aligned}
$$

Let

$$
y(t):=-\frac{\left(C_{p}+1\right)(p+1)}{p-1} J(u(t))+\frac{1}{2}\|u(t)\|_{H^{1}}^{2},
$$

then (3.12) implies

$$
y^{\prime}(t) \geq \frac{p-1}{C_{p}+1} y(t) .
$$

Calculating the above inequalities directly, we have

$$
\|u(t)\|_{H^{1}}^{2} \geq \frac{2\left(C_{p}+1\right)(p+1)}{p-1} J(u(t))+2 y(0) e^{\frac{p-1}{C_{p}+1} t}
$$

By condition (ii), we see

$$
y(0)=-\frac{\left(C_{p}+1\right)(p+1)}{p-1} J\left(u_{0}\right)+\frac{1}{2}\left\|u_{0}\right\|_{H^{1}}^{2}>0 .
$$

Recall that $J(u(t)) \geq 0$ and from (3.13), we deduce the inequality

$$
\|u(t)\|_{H^{1}} \geq(2 y(0))^{\frac{1}{2}} e^{\frac{p-1}{2\left(C_{p}+1\right)} t}
$$

which contradicts with (3.10) for $t$ sufficiently large. Thus $u(x, t)$ blows up at some finite time $t^{*}$.

Next, we give an upper bound estimate of $t^{*}$. For this, we firstly claim that

$$
I(u(t))=\int_{\Omega}|\nabla u|^{2} d x-\int_{\Omega}|u|^{p} u d x<0, \quad \forall t \in\left[0, t^{*}\right) .
$$


In fact, employing condition (ii) and Poincaré’s inequality (2.1), we obtain

$$
\begin{aligned}
I\left(u_{0}\right) & =(p+1) J\left(u_{0}\right)-\frac{p-1}{2}\left\|\nabla u_{0}\right\|_{2}^{2} \\
& <\frac{p-1}{2\left(C_{p}+1\right)}\left\|u_{0}\right\|_{H^{1}}^{2}-\frac{p-1}{2}\left\|\nabla u_{0}\right\|_{2}^{2} \\
& =\frac{p-1}{2\left(C_{p}+1\right)}\left\|u_{0}\right\|_{H^{1}}^{2}-\frac{C_{p}(p-1)}{2\left(C_{p}+1\right)}\left\|\nabla u_{0}\right\|_{2}^{2} \leq 0 .
\end{aligned}
$$

So, if (3.14) does not hold, there must exist $t_{1} \in\left(0, t^{*}\right)$ such that

$$
I\left(u\left(t_{1}\right)\right)=0, \quad I(u(t))<0, \quad \forall t \in\left[0, t_{1}\right) .
$$

Then, by $\frac{d}{d t}\left(\frac{1}{2}\|u(t)\|_{H^{1}}^{2}\right)=-I(u(t))$, we get $\|u(t)\|_{H^{1}}^{2}$ is strictly increasing on $\left[0, t_{1}\right)$, so it follows from condition (ii) that

$$
J\left(u_{0}\right)<\frac{p-1}{2(p+1)\left(C_{p}+1\right)}\left\|u_{0}\right\|_{H^{1}}^{2}<\frac{p-1}{2(p+1)\left(C_{p}+1\right)}\left\|u\left(t_{1}\right)\right\|_{H^{1}}^{2} .
$$

On the other hand, since $J(u(t))$ is nonincreasing with respect to $t$, we derive

$$
\begin{aligned}
J\left(u_{0}\right) \geq J\left(u\left(t_{1}\right)\right) & =\frac{1}{p+1} I\left(u\left(t_{1}\right)\right)+\frac{p-1}{2(p+1)}\left\|\nabla u\left(t_{1}\right)\right\|_{2}^{2} \\
& =\frac{p-1}{2(p+1)}\left\|\nabla u\left(t_{1}\right)\right\|_{2}^{2} \\
& =\frac{C_{p}(p-1)}{2(p+1)\left(C_{p}+1\right)}\left\|\nabla u\left(t_{1}\right)\right\|_{2}^{2}+\frac{p-1}{2(p+1)\left(C_{p}+1\right)}\left\|\nabla u\left(t_{1}\right)\right\|_{2}^{2} \\
& \geq \frac{p-1}{2(p+1)\left(C_{p}+1\right)}\left\|\nabla u\left(t_{1}\right)\right\|_{H^{1}}^{2},
\end{aligned}
$$

which contradicts with (3.15). Hence $I(u(t))<0$ and $\|u(t)\|_{H^{1}}^{2}$ is strictly increasing on $t \in$ $\left[0, t^{*}\right)$.

For any $\bar{T} \in\left(0, t^{*}\right)$, we define a functional by

$$
H(t):=\int_{0}^{t}\|u(\tau)\|_{H^{1}}^{2} d \tau+\left(t^{*}-t\right)\left\|u_{0}\right\|_{H^{1}}^{2}+\alpha(t+\beta)^{2}, \quad \forall t \in[0, \bar{T}]
$$

where $\alpha, \beta$ are two positive constants which will be determined later. Then

$$
H(0)=t^{*}\left\|u_{0}\right\|_{H^{1}}^{2}+\alpha \beta^{2}>0
$$

Since $\|u(t)\|_{H^{1}}^{2}$ is strictly increasing, we can write

$$
\begin{aligned}
H^{\prime}(t) & =\|u(t)\|_{H^{1}}^{2}-\left\|u_{0}\right\|_{H^{1}}^{2}+2 \alpha(t+\beta) \\
& =\int_{0}^{t} \frac{d}{d \tau}\|u(\tau)\|_{H^{1}}^{2} d \tau+2 \alpha(t+\beta) \\
& \geq 2 \alpha(t+\beta)>0 .
\end{aligned}
$$


Thus $H(t) \geq H(0)$ and $H(t)$ is strictly increasing on $[0, \bar{T}]$. Furthermore, applying (3.18) and (2.1), we deduce

$$
\begin{aligned}
H^{\prime \prime}(t) & =\frac{d}{d t}\|u(t)\|_{H^{1}}^{2}+2 \alpha=-2 I(u(t))+2 \alpha \\
& =(p-1)\|\nabla u(t)\|_{2}^{2}-2(p+1) J(u(t))+2 \alpha \\
& \geq \frac{p-1}{C_{p}+1}\left\|u_{0}\right\|_{H^{1}}^{2}-2(p+1) J\left(u_{0}\right)+2(p+1) \int_{0}^{t}\left\|u_{\tau}\right\|_{H^{1}}^{2} d \tau .
\end{aligned}
$$

By using the Cauchy-Schwarz inequality, Hölder's and Young's inequalities, we can derive

$$
\begin{aligned}
\xi(t):= & \left(\int_{0}^{t}\|u(\tau)\|_{H^{1}}^{2} d \tau+\alpha(t+\beta)^{2}\right)\left(\int_{0}^{t}\left\|u_{\tau}\right\|_{H^{1}}^{2} d \tau+\alpha\right) \\
& -\left(\frac{1}{2} \int_{0}^{t} \frac{d}{d \tau}\|u(\tau)\|_{H^{1}}^{2} d \tau+\alpha(t+\beta)\right)^{2} \geq 0, \quad \forall t \in[0, \bar{T}] .
\end{aligned}
$$

For any constant $\mu>0$, by (3.18)-(3.20), we have

$$
\begin{aligned}
H(t) H^{\prime \prime}(t)-\mu\left(H^{\prime}(t)\right)^{2} & \\
= & H(t) H^{\prime \prime}(t)-4 \mu\left(\frac{1}{2} \int_{0}^{t} \frac{d}{d \tau}\|u(\tau)\|_{H^{1}}^{2} d \tau+\alpha(t+\beta)\right)^{2} \\
= & H(t) H^{\prime \prime}(t)+4 \mu\left[\left(\int_{0}^{t}\|u(\tau)\|_{H^{1}}^{2} d \tau+\alpha(t+\beta)^{2}\right)\left(\int_{0}^{t}\left\|u_{\tau}\right\|_{H^{1}}^{2} d \tau+\alpha\right)\right] \\
& -4 \mu\left[H(t)-\left(t^{*}-t\right)\left\|u_{0}\right\|_{H^{1}}^{2}\right]\left(\int_{0}^{t}\left\|u_{\tau}\right\|_{H^{1}}^{2} d \tau+\alpha\right) \\
= & H(t) H^{\prime \prime}(t)+4 \mu \xi(t)-4 \mu\left[H(t)-\left(t^{*}-t\right)\left\|u_{0}\right\|_{H^{1}}^{2}\right]\left(\int_{0}^{t}\left\|u_{\tau}\right\|_{H^{1}}^{2} d \tau+\alpha\right) \\
\geq & H(t) H^{\prime \prime}(t)-4 \mu H(t)\left(\int_{0}^{t}\left\|u_{\tau}\right\|_{H^{1}}^{2} d \tau+\alpha\right) \\
\geq & H(t)\left[\frac{p-1}{C_{p}+1}\left\|u_{0}\right\|_{H^{1}}^{2}-2(p+1) J\left(u_{0}\right)+(2(p+1)-4 \mu) \int_{0}^{t}\left\|u_{\tau}\right\|_{H^{1}}^{2} d \tau-4 \mu \alpha\right] .
\end{aligned}
$$

Taking $\mu=\frac{p+1}{2}$ and restricting $\alpha$ to satisfy

$$
0<\alpha \leq \frac{p-1}{2(p+1)\left(C_{p}+1\right)}\left\|u_{0}\right\|_{H^{1}}^{2}-J\left(u_{0}\right)
$$

yields

$$
H(t) H^{\prime \prime}(t)-\frac{p+1}{2}\left(H^{\prime}(t)\right)^{2} \geq 0
$$

Let $W(t)=(H(t))^{\frac{1-p}{2}}$ for $t \in[0, \bar{T}]$, then by $H(t)>0, H^{\prime}(t)>0, p>1$, and (3.21), we obtain

$$
W^{\prime}(t)=\frac{1-p}{2}(H(t))^{-\frac{p+1}{2}} H^{\prime}(t)<0, \quad \forall t \in[0, \bar{T}],
$$


and

$$
W^{\prime \prime}(t)=\frac{1-p}{2}(H(t))^{-\frac{p+1}{2}-1}\left[H(t) H^{\prime \prime}(t)-\frac{p+1}{2}\left(H^{\prime}(t)\right)^{2}\right] \leq 0, \quad \forall t \in[0, \bar{T}]
$$

Then it follows from $W^{\prime \prime}(t) \leq 0$ that

$$
W(\bar{T})-W(0)=\int_{0}^{\bar{T}} W^{\prime}(t) d t \leq W^{\prime}(0) \bar{T}
$$

Since $W(0)=(H(0))^{\frac{1-p}{2}}>0$, it follows that

$$
\bar{T} \leq-\frac{W(0)}{W^{\prime}(0)}=\frac{\left\|u_{0}\right\|_{H^{1}}^{2}}{(p-1) \alpha \beta} t^{*}+\frac{\beta}{p-1}
$$

Hence, let $\bar{T} \rightarrow t^{*}$ and $\beta$ be large enough such that

$$
\frac{\left\|u_{0}\right\|_{H^{1}}^{2}}{(p-1) \alpha}<\beta<+\infty
$$

then we obtain

$$
t^{*} \leq \frac{\alpha \beta^{2}}{(p-1) \alpha \beta-\left\|u_{0}\right\|_{H^{1}}^{2}}
$$

Consider the function

$$
f(x, y):=\frac{x^{2}}{(p-1) x-\frac{\left\|u_{0}\right\|_{H^{1}}^{2}}{y}},
$$

where

$$
x \in\left(\frac{\left\|u_{0}\right\|_{H^{1}}^{2}}{(p-1) y},+\infty\right), \quad y \in\left(0, \frac{p-1}{2(p+1)\left(C_{p}+1\right)}\left\|u_{0}\right\|_{H^{1}}^{2}-J\left(u_{0}\right)\right] .
$$

For fixed $x$, we easily know $f(x, y)$ achieves its minimum at

$$
y=y_{0}:=\frac{p-1}{2(p+1)\left(C_{p}+1\right)}\left\|u_{0}\right\|_{H^{1}}^{2}-J\left(u_{0}\right)
$$

Through simple calculation, one can see that

$$
f^{\prime}\left(x, y_{0}\right)=\frac{x y_{0}\left((p-1) x y_{0}-2\left\|u_{0}\right\|_{H^{1}}^{2}\right)}{\left((p-1) x y_{0}-\left\|u_{0}\right\|_{H^{1}}^{2}\right)^{2}}, \quad x \in\left(\frac{\left\|u_{0}\right\|_{H^{1}}^{2}}{(p-1) y_{0}},+\infty\right)
$$

and $f\left(x, y_{0}\right)$ achieves its minimum at $x=x_{0}:=\frac{2\left\|u_{0}\right\|_{H^{1}}^{2}}{(p-1) y_{0}}$. Hence, it holds

$$
t^{*} \leq \frac{8(p+1)\left(C_{p}+1\right)\left\|u_{0}\right\|_{H^{1}}^{2}}{(p-1)^{2}\left[(p-1)\left\|u_{0}\right\|_{H^{1}}^{2}-2(p+1)\left(C_{p}+1\right) J\left(u_{0}\right)\right]} .
$$


In addition, if we make a further assumption for $p$ as follows:

$$
1<p<+\infty, \quad N=1,2 ; \quad 1<p \leq \frac{N+2}{N-2}, \quad N \geq 3,
$$

then, by (3.1) and Sobolev's embedding inequality from $H^{1}(\Omega)$ to $L^{p+1}(\Omega)$, we obtain

$$
\varphi^{\prime}(t) \leq 2 \int_{\Omega}|u|^{p} u d x \leq 2 C_{s}^{p+1}\left(\int_{\Omega}|\nabla u|^{2} d x\right)^{\frac{p+1}{2}} \leq 2 C_{s}^{p+1}(\varphi(t))^{\frac{p+1}{2}}
$$

where $C_{s}=C_{s}(\Omega, p)$ is the optimal embedding constant. Integrating (3.23) from 0 to $t$, we derive

$$
\varphi(t) \leq\left[(\varphi(0))^{-\frac{p-1}{2}}-C_{s}^{p+1}(p-1) t\right]^{-\frac{2}{p-1}} .
$$

Therefore, we have

$$
t^{*} \geq \frac{1}{C_{s}^{p+1}(p-1)\left\|u_{0}\right\|_{H^{1}}^{p-1}} .
$$

This completes the proof.

Proof of Corollary 1 Let $\Omega_{1}, \Omega_{2}$ be such that

$$
\Omega_{1}, \Omega_{2} \subset \Omega, \quad \Omega_{1} \cap \Omega_{2}=\varnothing,
$$

and let $v \in C_{0}\left(\Omega_{1}\right) \cap H$ be an arbitrary nonzero function. Suppose $J\left(u_{0}\right)=R>0$, then there exists $r_{1}$ sufficiently large to satisfy

$$
\left\|r_{1} v\right\|_{H_{0}^{1}(\Omega)}^{2}=r_{1}^{2}\|v\|_{H_{0}^{1}\left(\Omega_{1}\right)}^{2}>\frac{2(p+1)\left(C_{p}+1\right)}{p-1} R .
$$

We claim that there exist $\bar{\omega} \in H$ and $r>r_{1}$ such that $J(\bar{\Omega})=R-J(r v)$. By the similar argument in [33, Theorem 1.1], we know there exists a sequence $\left\{\omega_{k}\right\} \subset C_{0}\left(\Omega_{2}\right) \cap H$ such that

$$
\frac{1}{2} \int_{\Omega}\left|\nabla \omega_{k}\right|^{2} d x-\frac{1}{p+1} \int_{\Omega}\left|\omega_{k}\right|^{p} \omega_{k} d x \rightarrow+\infty, \quad \text { as } k \rightarrow \infty .
$$

On the other hand, since $p>1$, it holds

$$
R-J(r v)=R-\frac{r^{2}}{2} \int_{\Omega}|\nabla v|^{2} d x+\frac{r^{p+1}}{p+1} \int_{\Omega}|v|^{p} v d x \rightarrow+\infty, \quad \text { as } k \rightarrow \infty .
$$

So, there exist $k \in \mathbb{N}$ and $r>r_{1}$ both sufficiently large such that

$$
R-J(r v)=\frac{1}{2} \int_{\Omega}\left|\nabla \omega_{k}\right|^{2} d x-\frac{1}{p+1} \int_{\Omega}\left|\omega_{k}\right|^{p} \omega_{k} d x
$$


Now, we choose

$$
\bar{\omega}= \begin{cases}\omega_{k}, & x \in \Omega_{2}, \\ 0, & x \in \Omega \backslash \Omega_{2} .\end{cases}
$$

Then, for $u_{R}:=r v+\bar{\omega}$, we have

$$
\begin{aligned}
J\left(u_{R}\right) & =J(r v)+J(\bar{\omega})=R<\frac{p-1}{2(p+1)\left(C_{p}+1\right)}\left\|r_{1} v\right\|_{H_{0}^{1}(\Omega)}^{2} \\
& <\frac{p-1}{2(p+1)\left(C_{p}+1\right)}\left\|r_{1} v+\bar{\omega}\right\|_{H^{1}(\Omega)}^{2}=\frac{p-1}{2(p+1)\left(C_{p}+1\right)}\left\|u_{R}\right\|_{H^{1}(\Omega)}^{2} .
\end{aligned}
$$

According to Theorem 1, the solution $u(x, t)$ of problem (1.1)-(1.3) with initial data $u_{R}$ blows up at finite time. This completes the proof.

For the proof of Theorem 2, we first state a critical lemma.

Lemma 1 ([34] ) Suppose that $\gamma, \theta, \eta>0$ and $\zeta(t)$ is a nonnegative and absolutely continuous function satisfying

$$
\zeta^{\prime}(t)+\gamma \zeta^{\theta}(t) \geq \eta, \quad 0<t<\infty
$$

then

$$
\zeta(t) \geq \min \left\{\zeta(0),\left(\frac{\eta}{\gamma}\right)^{\frac{1}{\theta}}\right\}
$$

Proof of Theorem 2 Set

$$
z_{1}(x, t):=u(x, t)-\left(\left\|u_{0}\right\|_{L^{\infty}(\Omega)}+t\|u\|_{L^{\infty}\left(\Omega \times\left(0, t^{*}\right)\right)}^{p}\right) .
$$

A direct computation shows

$$
\left\{\begin{array}{l}
z_{1 t}-\Delta z_{1 t}-\Delta z_{1} \leq 0, \quad(x, t) \in \Omega \times\left(0, t^{*}\right) \\
\frac{\partial z_{1}}{\partial n}=0, \quad(x, t) \in \partial \Omega \times\left(0, t^{*}\right) \\
z_{1}(x, 0) \leq 0, \quad x \in \Omega
\end{array}\right.
$$

By the weak maximum principle [35], we have $z_{1}(x, t) \leq 0$, namely

$$
u(x, t) \leq\left\|u_{0}\right\|_{L^{\infty}(\Omega)}+t\|u\|_{L^{\infty}\left(\Omega \times\left(0, t^{*}\right)\right)}^{p} .
$$

Similarly, set

$$
z_{2}(x, t):=-u(x, t)-\left(\left\|u_{0}\right\|_{L^{\infty}(\Omega)}+t\|u\|_{L^{\infty}\left(\Omega \times\left(0, t^{*}\right)\right)}^{p}\right)
$$

we can obtain the boundedness from below

$$
u(x, t) \geq-\left(\left\|u_{0}\right\|_{L^{\infty}(\Omega)}+t\|u\|_{L^{\infty}\left(\Omega \times\left(0, t^{*}\right)\right)}^{p}\right) .
$$


Thus, it follows from (3.25) and (3.26) that

$$
\sup _{t \in\left(0, t^{*}\right)}\|u(t)\|_{L^{\infty}(\Omega)} \leq\left\|u_{0}\right\|_{L^{\infty}(\Omega)}+t\|u\|_{L^{\infty}\left(\Omega \times\left(0, t^{*}\right)\right)}^{p}
$$

which implies that the solution $u(x, t)$ of problem (1.1)-(1.3) is global whenever $p<1$.

Furthermore, we impose the condition $\left\|u_{0}\right\|_{H^{1}}^{2}>\frac{\left(C_{p}+1\right) \chi(\varepsilon)|\Omega|}{2-C_{p} \varepsilon}$, where $0<\varepsilon<\frac{2}{C_{p}}$ and $\chi(\varepsilon)=\frac{1-p}{2}\left(\frac{p+1}{\varepsilon}\right)^{\frac{1}{1-p}}$. Firstly, since $p<1$, we can derive the following inequality by virtue of Hölder's and Young's inequalities:

$$
\begin{aligned}
2 \int_{\Omega}|u|^{p} u d x & \leq 2\left(\int_{\Omega}|u|^{2} d x\right)^{\frac{p+1}{2}}|\Omega|^{\frac{1-p}{2}} \\
& \leq \varepsilon \int_{\Omega}|u|^{2} d x+\chi(\varepsilon)|\Omega|
\end{aligned}
$$

Then, by (2.1), we obtain

$$
\begin{aligned}
-2 \int_{\Omega}|\nabla u|^{2} d x & =-\frac{C_{p}(2+\varepsilon)}{C_{p}+1} \int_{\Omega}|\nabla u|^{2} d x-\frac{2-C_{p} \varepsilon}{C_{p}+1} \int_{\Omega}|\nabla u|^{2} d x \\
& \leq-\frac{2+\varepsilon}{C_{p}+1} \int_{\Omega}|u|^{2} d x-\frac{2-C_{p} \varepsilon}{C_{p}+1} \int_{\Omega}|\nabla u|^{2} d x
\end{aligned}
$$

Afterwards, substituting (3.27) and (3.28) into (3.1), we deduce

$$
\varphi^{\prime}(t) \leq-\frac{2-C_{p} \varepsilon}{C_{p}+1} \varphi(t)+\chi(\varepsilon)|\Omega|
$$

Integrating (3.29) from 0 to $t$, it yields

$$
\varphi(t) \leq\left(\varphi(0)-\frac{\left(C_{p}+1\right) \chi(\varepsilon)|\Omega|}{2-C_{p} \varepsilon}\right) e^{-\frac{2-C_{p} \varepsilon}{C_{p}+1} t}+\frac{\left(C_{p}+1\right) \chi(\varepsilon)|\Omega|}{2-C_{p} \varepsilon}
$$

Hence, it is easy to see that the solution $u(x, t)$ of problem (1.1)-(1.3) decays in the sense of $H^{1}(\Omega)$-norm when $\varphi(0)>\frac{\left(C_{p}+1\right) \chi(\varepsilon)|\Omega|}{2-C_{p} \varepsilon}$, and we have

$$
\|\left. u(t)\right|_{H^{1}} ^{2} \leq\left(\left\|u_{0}\right\|_{H^{1}}^{2}-\frac{\left(C_{p}+1\right) \chi(\varepsilon)|\Omega|}{2-C_{p} \epsilon}\right) \exp \left\{-\frac{2-C_{p} \varepsilon}{C_{p}+1} t\right\}+\frac{\left(C_{p}+1\right) \chi(\varepsilon)|\Omega|}{2-C_{p} \varepsilon}
$$

Finally, we claim that the solution $u(x, t)$ is positive in the sense of $W^{1, s}(\Omega)$-norm for any $s>1$ under $J\left(u_{0}\right)<0$. To this end, applying (2.2) and (3.1), we get

$$
\begin{aligned}
\frac{1}{2} \varphi^{\prime}(t) & =-I(u(t))=-2 J(u(t))-\frac{1-p}{1+p} \int_{\Omega}|u|^{p} u d x \\
& =-2 J\left(u_{0}\right)+2 \int_{0}^{t}\left\|u_{\tau}\right\|_{H^{1}}^{2} d \tau-\frac{1-p}{1+p} \int_{\Omega}|u|^{p} u d x \\
& \geq-2 J\left(u_{0}\right)-\frac{1-p}{1+p} \int_{\Omega}|u|^{p} u d x
\end{aligned}
$$


Since $p<1$, utilizing Hölder's inequality yields

$$
\begin{aligned}
\int_{\Omega}|u|^{p} u d x & \leq\left(\int_{\Omega}|u|^{2} d x\right)^{\frac{p+1}{2}}|\Omega|^{\frac{1-p}{2}} \\
& \leq\left(\int_{\Omega}|u|^{2}+|\nabla u|^{2} d x\right)^{\frac{p+1}{2}}|\Omega|^{\frac{1-p}{2}} \\
& =(\varphi(t))^{\frac{p+1}{2}}|\Omega|^{\frac{1-p}{2}} .
\end{aligned}
$$

Substituting (3.31) into (3.30), we deduce

$$
\varphi^{\prime}(t) \geq-A(\varphi(t))^{\frac{p+1}{2}}-4 J\left(u_{0}\right)
$$

where $A=\frac{2(1-p)}{1+p}|\Omega|^{\frac{1-p}{2}}$. By Lemma 1 and the fact $J\left(u_{0}\right)<0$, there holds

$$
\varphi(t) \geq \min \left\{\varphi(0),\left(\frac{-4 J\left(u_{0}\right)}{A}\right)^{\frac{2}{p+1}}\right\}, \quad t>0 .
$$

Since $\varphi(0)=\left\|u_{0}\right\|_{H^{1}}^{2}>0, A>0, J\left(u_{0}\right)<0$, we get $\varphi(t)>0, \forall t>0$. For any $s>1$, by the interpolation inequality, we obtain

$$
\|u\|_{2}^{2} \leq\|u\|_{s}\|u\|_{s^{\prime}}, \quad\|\nabla u\|_{2}^{2} \leq\|\nabla u\|_{s}\|\nabla u\|_{s^{\prime}},
$$

where $\frac{1}{s}+\frac{1}{s^{\prime}}=1$. Then we can deduce that

$$
\begin{aligned}
\|u\|_{H^{1}}^{2} & \leq\|u\|_{s}\|u\|_{s^{\prime}}+\|\nabla u\|_{s}\|\nabla u\|_{s^{\prime}} \\
& \leq 2\left(\|u\|_{s}^{s}+\|\nabla u\|_{s}^{s}\right)^{\frac{1}{s}}\left(\|u\|_{s^{\prime}}^{s^{\prime}}+\|\nabla u\|_{s^{\prime}}^{s^{\prime}}\right)^{\frac{1}{s^{\prime}}} \\
& =2\|u\|_{W^{1, s}}\|u\|_{W^{1, s^{\prime}}},
\end{aligned}
$$

which combined with $\|u\|_{H^{1}}^{2}>0$ implies that, for every $s>1$, there does not exist $T^{*}>0$ such that

$$
\lim _{t \rightarrow T^{*}}\|u\|_{W^{1, s}}=0
$$

This completes the proof.

Proof of Theorem 3 Let $p=1$ in the proof of Theorem 1(i), we have

$$
\psi(t)=\varphi^{\prime}(t), \quad t \in\left(0, t^{*}\right)
$$

According to (3.7), we get

$$
\frac{\varphi^{\prime}(t)}{\varphi(t)}>\frac{\psi(0)}{\varphi(0)}, \quad t \in\left(0, t^{*}\right)
$$


Integrating (3.32) from 0 to $t$, it follows that

$$
\varphi(t) \geq \varphi(0) e^{\frac{\psi(0)}{\varphi(0)} t}, \quad t \in\left(0, t^{*}\right) .
$$

Thus, the solution $u(x, t)$ to problem (1.1)-(1.3) grows within $\left(0, t^{*}\right)$ in the sense of $H^{1}(\Omega)$ norm, and we have

$$
\|u(t)\|_{H^{1}}^{2} \geq\left\|u_{0}\right\|_{H^{1}}^{2} \exp \left\{\frac{-4 J\left(u_{0}\right)}{\left\|u_{0}\right\|_{H^{1}}^{2}} t\right\} .
$$

This completes the proof.

\section{Acknowledgements}

The authors would like to deeply thank all the reviewers for their insightful and constructive comments.

\section{Funding}

This work is supported by the National Natural Science Foundation of China (No. 11671188) and the Fundamental Research Funds for the Central Universities (Grant No. 201964008).

\section{Abbreviations}

Not applicable.

\section{Availability of data and materials}

Data sharing not applicable to this article as no datasets were generated or analysed during the current study.

\section{Competing interests}

The authors declare that there is no conflict of interests regarding the publication of this paper.

\section{Authors' contributions}

All authors contributed equally and significantly in writing this article. All authors read and approved the final manuscript.

\section{Publisher's Note}

Springer Nature remains neutral with regard to jurisdictional claims in published maps and institutional affiliations.

Received: 13 February 2019 Accepted: 30 July 2019 Published online: 09 August 2019

\section{References}

1. Barenblat, G., Zheltov, I., Kochiva, I.: Basic concepts in the theory of seepage of homogeneous liquids in fissured rocks. J. Appl. Math. Mech. 24, 1286-1303 (1960)

2. Benjamin, T.B., Bona, J.L., Mahony, J.J.: Model equations for long waves in nonlinear dispersive systems. Philos. Trans. R. Soc. Lond. Ser. A 272, 47-78 (1972)

3. Ting, T.W.: Certain non-steady flows of second-order fluids. Arch. Ration. Mech. Anal. 14, 1-26 (1963)

4. Chen, P.J., Gurtin, M.E.: On a theory of heat conduction involving two temperatures. Z. Angew. Math. Phys. 19 614-627 (1968)

5. Korpusov, M.O., Sveshnikov, A.G.: Three-dimensional nonlinear evolution equations of pseudoparabolic type in problems of mathematical physics. Zh. Vychisl. Mat. Mat. Fiz. 43, 1835-1869 (2003)

6. Furter, J., Grinfield, M.: Local vs. non-local interactions in population dynamics. J. Math. Biol. 27, 65-80 (1989)

7. Calsina, A., Perello, C., Saldana, J.: Non-local reaction-diffusion equations modelling predator-prey coevolution. Publ. Mat. 38, 315-325 (1994)

8. Padron, V:: Effect of aggregation on population recovery modeled by a forward-backward pseudoparabolic equation. Trans. Am. Math. Soc. 356, 2739-2756 (2004)

9. Allegretto, W., Fragnelli, G., Nistri, P., Papin, D.: Coexistence and optimal control problems for a degenerate predator-prey mode. J. Math. Anal. Appl. 378, 528-540 (2011)

10. Lorz, A., Mirrahimi, S., Perthame, B.: Dirac mass dynamics in multidimensional nonlocal parabolic equations. Commun. Partial Differ. Equ. 36, 1071-1098 (2011)

11. Lorz, A., Lorenzi, T., Clairambault, J., Escargueil, A., Perthame, B.: Effects of space structure and combination therapies on phenotypic heterogeneity and drug resistance in solid tumors. Bull. Math. Biol. 77, 1-22 (2013)

12. Volpert, V., Vougalter, V.: Existence of stationary pulses for nonlocal reaction-diffusion equations. Doc. Math. 19 1141-1153 (2014)

13. Budd, C., Dold, B., Stuart, A.: Blow up in a partial differential equation with conserved first integral. SIAM J. Appl. Math. 53, 718-742 (1993)

14. Budd, C., Dold, B., Stuart, A.: Blow-up in a system of partial differential equations with conserved first integral. II. Problems with convection. SIAM J. Appl. Math. 54, 610-640 (1994)

15. Hu, B., Yin, H.M.: Semilinear parabolic equations with prescribed energy. Rend. Circ. Mat. Palermo 44, 479-505 (1995) 
16. Gao, W., Han, Y.: Blow-up of a nonlocal semilinear parabolic equation with positive initial energy. Appl. Math. Lett. 24, 784-788 (2011)

17. El Soufi, A., Jazar, M., Monneau, R.: A gamma-convergence argument for the blow-up of a non-local semilinear parabolic equation with Neumann boundary conditions. Ann. Inst. Henri Poincaré, Anal. Non Linéaire 24, 17-39 (2007)

18. Jazar, M., Kiwan, R.: Blow-up of a non-local semilinear parabolic equation with Neumann boundary conditions. Ann. Inst. Henri Poincaré, Anal. Non Linéaire 25, 215-218 (2008)

19. Niculescu, C.P., Roventa, J.: Large solutions for semilinear parabolic equations involving some special classes of nonlinearities. Discrete Dyn. Nat. Soc. 2010, Article ID 491023 (2010)

20. Wang, X.L., Tian, F.Z., Li, G.: Nonlocal parabolic equation with conserved spatial integral. Arch. Math. 105, 93-100 (2015)

21. Wang, H.L., Tao, W.R., Wang, X.L.: Finite-time blow-up and global convergence of solutions to a nonlocal parabolic equation with conserved spatial integral. Nonlinear Anal., Real World Appl. 40, 55-63 (2018)

22. Yan, L., Yang, Z: Blow-up and non-extinction for a nonlocal parabolic equation with logarithmic nonlinearity. Bound. Value Probl. 2018, 121 (2018)

23. Xu, G.Y., Zhou, J.: Global existence and finite time blow-up of the solution for a thin-film equation with high initial energy. J. Math. Anal. Appl. 458, 521-535 (2018)

24. Cao, Y., Liu, C.H.: Global existence and non-extinction of solutions to a fourth-order parabolic equation. Appl. Math. Lett. 61, 20-25 (2016)

25. Niculescu, C.P., Roventa, J.: Generalized convexity and the existence of finite time blow-up solutions for an evolutionary problem. Nonlinear Anal. 75, 270-277 (2012)

26. Fang, Z.B., Sun, L., Li, C.J.: A note on blow-up of solutions for the nonlocal quasilinear parabolic equation with positive initial energy. Bound. Value Probl. 2013, 181 (2013)

27. Hao, A.J., Zhou, J.: Blowup, extinction and non-extinction for a nonlocal p-biharmonic parabolic equation. Appl. Math. Lett. 64, 198-204 (2017)

28. Cao, Y., Yin, J.X., Wang, C.P.: Cauchy problems of semilinear pseudo-parabolic equations. J. Differ. Equ. 246, 4568-4590 (2009)

29. Xu, R.Z., Su, J.: Global existence and finite time blow-up for a class of semilinear pseudo-parabolic equations. J. Funct. Anal. 264, 2732-2763 (2013)

30. Luo, P.: Blow-up phenomena for a pseudo-parabolic equation. Math. Methods Appl. Sci. 38, 2636-2641 (2015)

31. Xu, G.Y., Zhou, J.: Lifespan for a semilinear pseudo-parabolic equation. Math. Methods Appl. Sci. 41, 705-713 (2018)

32. Yang, L., Liang, F.: Bounds for blow-up time in a semilinear pseudo-parabolic equation with nonlocal source. J. Inequal. Appl. 2016, 229 (2016)

33. Sun, F., Liu, L., Wu, Y.: Infinitely many sign-changing solutions for a class of biharmonic equation with $p$-Laplacian and Neumann boundary condition. Appl. Math. Lett. 73, 28-135 (2017)

34. Guo, B., Gao, W.J.: Non-extinction of solutions to a fast diffusive p-Laplace equation with Neumann boundary conditions. J. Math. Anal. Appl. 422, 1527-1531 (2015)

35. Stecher, M., Rundell, W.: Maximum principles for pseudoparabolic partial differential equations. J. Math. Anal. Appl. $57,110-118(1977)$

\section{Submit your manuscript to a SpringerOpen ${ }^{\circ}$ journal and benefit from:}

- Convenient online submission

- Rigorous peer review

- Open access: articles freely available online

- High visibility within the field

- Retaining the copyright to your article

Submit your next manuscript at $\gg$ springeropen.com 